\title{
Zur Bedeutung des Freihandels und globaler Wertschöpfungsketten für Deutschland und Österreich
}

\author{
Wolfgang Schwarzbauer (iD) P Philipp Koch $(\mathbb{D}$
}

Angenommen: 21. April 2021 / Online publiziert: 20. Mai 2021

(C) List-Gesellschaft e.V. 2021

Zusammenfassung Vielerorts wurde aufgrund der COVID-19 Pandemie hinterfragt, ob die derzeitige globale wirtschaftliche Vernetzung weiterhin wünschenswert sei, und ob nicht eine Re-Regionalisierung der Produktion angestrebt werden sollte. Im Rahmen dieses Beitrags erörtern wir deshalb die Bedeutung des Freihandels, im Speziellen der ausländischen Endnachfrage, für die Wertschöpfung in Deutschland und Österreich. Es zeigt sich, dass sowohl Deutschland als auch Österreich eng in globale Wertschöpfungsketten integriert sind und eine vergleichsweise zentrale Position einnehmen. Darüber hinaus wird deutlich, dass rund ein Drittel der Wertschöpfung in Deutschland und Österreich schließlich im Ausland konsumiert oder investiert wird. In Industriebranchen ist der Anteil der im Ausland der Endnachfrage zugeführten Wertschöpfung deutlich höher und beträgt in einzelnen Branchen über $80 \%$. Auch für einzelne im Außenhandel wichtige Dienstleistungsbranchen wie dem Großhandel oder Finanzdienstleistungen ist die globale Verflechtung zentral. Aufgrund der hohen volkswirtschaftlichen Kosten, die mit einer Re-Regionalisierung aufgrund von möglichen Vergeltungsmaßnahmen sowie möglichen mittelfristigen Verlusten von Spezialisierungsvorteilen einhergehen, sollte die COVID-19 Pandemie nicht als Anlass für eine Rückkehr zu einer protektionistischeren Handelspolitik herangezogen werden. Vielmehr soll das Ziel der Wirtschaftspolitik sein, die Rolle deutscher und österreichischer Unternehmen im Außenhandel und globalen Wertschöpfungsketten durch eine Steigerung der Produktivität zu stärken und eine Weiterentwicklung der europäischen und weltweiten Integration voranzutreiben.

Schlüsselwörter Freihandel · Globale Wertschöpfungsketten · COVID-19

\footnotetext{
W. Schwarzbauer $\cdot$ P. Koch $(\bowtie)$

EcoAustria - Institut für Wirtschaftsforschung, Wien, Österreich

E-Mail: philipp.koch@ecoaustria.ac.at

W. Schwarzbauer

E-Mail: wolfgang.schwarzbauer@ecoaustria.ac.at
} 


\title{
On the significance of free trade and global value chains for Germany and Austria
}

\begin{abstract}
During the COVID-19 pandemic, global value chain integration became increasingly questioned and the idea of reshoring the production gained momentum. In this contribution, we discuss the importance of free trade and value chain integration with special focus on the role of global final demand for Austria and Germany. We show that Germany and Austria are deeply integrated in global value chains and hold a relatively central position. Furthermore, roughly a third of value added of the two countries is eventually consumed or invested abroad. While the share of value added consumed or invested abroad accrues to more than $80 \%$ in some manufacturing industries, specific services industries are also deeply integrated in global value chains, most notably wholesale trade and financial services. Nevertheless, most services industries' value added is predominantly consumed or invested domestically. Due to large economic welfare costs of reshoring global value chains, specifically because of retaliation measures as well as potential losses of comapartive advantages, the COVID-19 pandemic should not be used as a justification for a return to a more protectionist international trade regime. Thus, it is central for economic policy in Austria and Germany to support and promote the presence of their companies in international value chains by fostering their productivity and to spur the advancement of European and global economic integration.
\end{abstract}

Keywords Free trade $\cdot$ Global value chains $\cdot$ COVID-19

\section{Hintergrund und Motivation}

Die COVID-19 Krise, ausgelöst durch den Ausbruch der Pandemie und der als Reaktion eingeleiteten Maßnahmen der Regierungen vieler Staaten in Form von Lockdowns und anderer Beschränkungen, hat dramatische Auswirkungen auf die globale Wirtschaft und den internationalen Handel.

Bei unterschiedlichen Artikeln und vor allem bei Schutzausrüstung für medizinisches Personal kam es im Zuge der Pandemie zu Engpässen. Dies offenbarte wie stark vernetzt und spezialisiert die internationale Produktion bereits ist. Als Reaktion darauf versuchten viele Staaten der Welt die Ausfuhr von auf ihrem Territorium befindlichen Beständen an notwendiger medizinischer Ausrüstung zu verhindern oder zumindest zu beschränken, wie etwa die USA Anfang April 2020. Aber auch in den EU-Staaten wurden verstärkt derartige Maßnahmen erwogen. Bereits Anfang März wurde in Frankreich verfügt, alle Bestände an medizinischen Masken und Beatmungsgeräten auf französischen Boden zu konfiszieren (Fiorini et al. 2020). Vor diesem Hintergrund versuchten viele Staaten entsprechende nationale Produktionskapazitäten aufzubauen. Weiters wurde hinterfragt, ob die globale Vernetzung, wie sie sich Anfang 2020 darstellte, noch wünschenswert sei oder ob die globale Produktion wieder re-regionalisiert werden sollte. Eine Desintegration globaler Wert- 


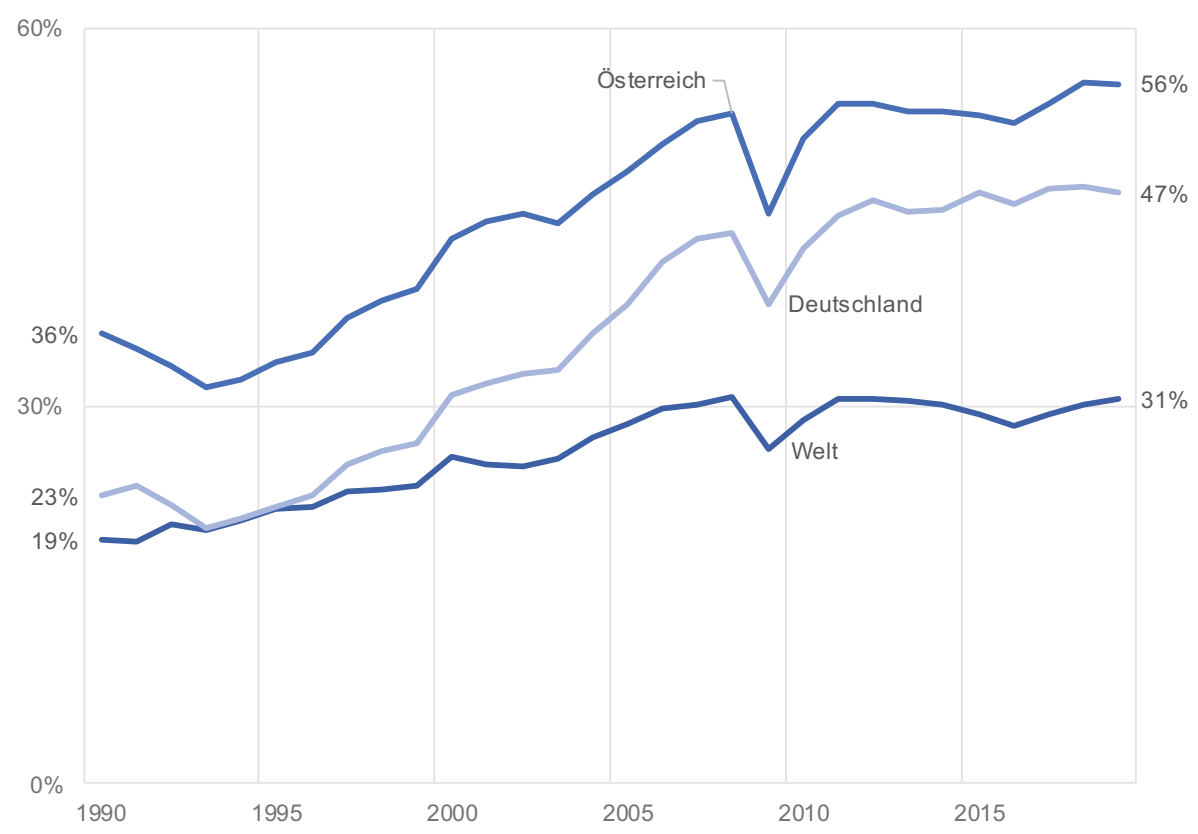

Abb. 1 Bruttoexporte in Relation zum BIP, Österreich, Deutschland und Welt, 1990-2019. (Quelle: World Bank Indicators)

schöpfungsketten beeinflusst dabei nicht nur die Vorleistungsmärkte der deutschen und österreichischen Volkswirtschaften, sondern auch deren Weiterverarbeitungsund Endnachfrageverflechtungen.

Sowohl Deutschland als auch Österreich haben sich in den letzten Jahrzehnten in den internationalen Waren- und Dienstleistungshandel integriert. Abb. 1 zeigt die Bruttoexporte (Waren und Dienstleistungen) der beiden Länder und der Welt in Relation zum jeweiligen Bruttoinlandsprodukt (BIP). Über die vergangenen dreißig Jahre erhöhte sich dieses Verhältnis in Deutschland von 23\% im Jahr 1990 auf 47\% im Jahr 2019 und im Falle Österreichs von 38\% im Jahr 1990 auf 57\% im Jahr 2019. Zwischen 1993 und der Wirtschafts- und Finanzkrise 2009 entwickelte sich dieses Verhältnis in Deutschland und Österreich äußerst dynamisch und lag nach 2009 auch sehr rasch auf Vorkrisenniveau. Bemerkenswert ist vor allem die deutlich positivere Entwicklung des Außenhandels in Deutschland und Österreich im Vergleich zur weltweiten Handelsintensität. Insbesondere der Fall des Eisernen Vorhangs, aber auch einige EU-Erweiterungen, sowie die Digitalisierung und fallende Transport- und Handelskosten trugen zu der positiven Entwicklung bis 2009 bei. Zudem trug der sich in Mitteleuropa bildende Central European Manufacturing Core mit Deutschland als Technologiegeber, den Visegrád-Staaten als Technologienehmern sowie Österreich als Verbindungsglied zu der positiven Exportentwicklung von Deutschland und Österreich bei. Gerade in der Wirtschafts- und Finanzkrise 2009 erwies sich dieser Produktionsverbund als resilient und erholte sich rasche als andere europäische Produktionsnetzwerke (siehe Stehrer und Stöllinger 2015). 
Seit 2009 ist aber sowohl weltweit als auch in Deutschland und Österreich eine Seitwärtsbewegung des Außenhandels in Prozent des BIP zu erkennen.

Die Globalisierung und die zunehmenden Handelsverflechtungen haben sich für Deutschland und Österreich auch positiv auf das Pro-Kopf-Einkommen ausgewirkt. So zeigen Felbermayr et al. (2018), dass die Pro-Kopf-Einkommen ohne einer EUMitgliedschaft auch nach Berücksichtigung der Nettozahlungen um 5,1 (Deutschland) bzw. 7,9\% (Österreich) niedriger ausfielen. Auch laut aktuellem Globalisierungsreport der Bertelsmann-Stiftung (vgl. Sachs et al. 2020) zählen Deutschland und Österreich zu jenen Ländern, die maßgeblich von der Globalisierung profitierten: So liegt das Pro-Kopf-Einkommen aufgrund der vornaschreitenden Globalisierung seit 1990 den Berechnungen der Bertelsmann-Stiftung zufolge im Jahr 2018 um 4,6 (Deutschland) bzw. 3,1\% (Österreich) höher. Zu ähnlich positiven Ergebnissen der Globalisierung sowie konkret der der EU-Mitgliedschaft kommen Breuss (2020), Mayer et al. (2019) sowie Berger et al. (2014).

Mit dem Anstieg des Handelsvolumens zwischen 1990 und 2009 ging zudem die vermehrte Bedeutung von globalen Wertschöpfungsketten einher (Weltbank 2019). Die Produktion von Gütern wurde stets feingliedriger, sodass sich einzelne Länder auf sehr konkrete Produktionsschritte spezialisieren konnten. Nach 2009 erfuhr der Handel in globalen Wertschöpfungsketten aufgrund protektionistischer Tendenzen in verschiedenen Teilen der Welt einen leichten Rückgang. So kam es nach $2009 \mathrm{zu}$ einer Verkürzung globaler Wertschöpfungsketten (Felbermayr und Görg 2020). Speziell chinesische Unternehmen haben die Interdependenzen in Wertschöpfungsketten signifikant reduziert (Flach et al. 2020).

Der vorliegende Beitrag zeigt zum einen auf, inwieweit Deutschland und Österreich in globale Wertschöpfungsketten eingebunden sind. Zum anderen wird dargestellt wie bedeutend die ausländische Endnachfrage für die heimische Wertschöpfung in beiden Volkswirtschaften bereits geworden ist. Unter ausländischer Endnachfrage werden dabei der Konsum und die Investitionen in anderen Ländern verstanden. Eine vergleichbare Analyse der Weltwirtschaftsverflechtungen Deutschlands haben jüngst Flach et al. (2020) unternommen, die ähnliche Ergebnisse für Deutschland zeigt. In diesem Beitrag verwenden wir eine andere Datenbasis und betrachten damit die einzelnen Branchen detaillierter. Darüber hinaus ergibt der in diesem Beitrag dargelegte Vergleich zwischen Deutschland und Österreich, dass die Ergebnisse nicht spezifisch für Deutschland gelten, sondern auch für andere EU-Mitgliedsstaaten ähnlich sind.

Der Beitrag ist wie folgt strukturiert: In Kapitel 2 wird Deutschlands und Österreichs Rolle in globalen Wertschöpfungsketten beleuchtet, während die internationalen Verflechtungen der beiden Volkswirtschaften mit der ausländischen Endnachfrage auf Branchenebene in Kapitel 3 dargestellt werden. Kapitel 4 schließt mit wirtschaftspolitischen Implikationen ab.

\section{Die Rollen Deutschlands und Österreichs in globalen Wertschöpfungsketten}

Globale Wertschöpfungsketten können als eine Verbindung von aufeinander aufbauenden Produktionsschritten gesehen werden. Diese reichen von der Rohstoffgewin- 
nung sowie der Produktentwicklung, über die tatsächlichen einzelnen Produktionsschritte der Zwischengüter und des Endprodukts bis hin zum Vertrieb.

Zur Darstellung der Verflechtung in globalen Wertschöpfungsketten sind insbesondere zwei Kennzahlen relevant. Zum einen ist das die Partizipation an Wertschöpfungsketten. Darunter wird verstanden wie groß der Anteil des Handels in Wertschöpfungsketten, sowohl import- als auch exportseitig, am gesamten Außenhandel der jeweiligen Branche oder des jeweiligen Landes ist. Zum anderen wird die Position in Wertschöpfungsketten beschrieben. Die Position orientiert sich am Verhältnis von vorgelagerten zu nachgelagerten Produktionsschritten. Überwiegen aus der Sicht eines spezifischen Landes oder einer spezifischen Branche die nachgelagerten Produktionsschritte, so wird die Position als „upstream“ beschrieben, d.h. am Beginn einer Wertschöpfungskette. Das ist beispielsweise in der Rohstoffgewinnung oder der Forschung \& Entwicklung der Fall. Im Gegensatz dazu wird von ,downstream“, d.h. am Ende einer Wertschöpfungskette, gesprochen, wenn die vorgelagerten Produktionsschritte überwiegen, z. B. im Groß- oder Einzelhandel.

Dabei ist zu erwähnen, dass es a priori nicht möglich ist festzustellen, ob eine Einordnung in ,downstream“ oder ,upstream“ zu bevorzugen ist. Vielmehr ist dies markant von der Wertschöpfungsstruktur der betrachteten Branche und den Spezifika der jeweiligen Wertschöpfungskette abhängig. Setzt man die Position in der Wertschöpfungskette mit der Entlohnung pro Arbeitsstunde in Verbindung, so zeigt sich beispielsweise für chinesische Exporte in der Branche „Computer, Elektronik und Optik“ ein U-förmiger Zusammenhang (sog. „smile curve“). Die Entlohnung ist somit an den beiden Enden der Wertschöpfungskette am höchsten, d.h. bei der Entwicklung des Produkts (,upstream“), sowie beim Vertrieb (,downstream“). Die tatsächliche Produktion hat hingegen eine geringere Entlohnung. Anders stellt sich dies bei deutschen Exporten der Automobilindustrie dar. Dort ist die Entlohnung pro Arbeitsstunde in der Produktion vor allem in Deutschland, d.h. in der Mitte der Wertschöpfungskette, am höchsten (vgl. IBRD 2017, S. 52ff.).

Im Rahmen dieses Beitrags wird auf Daten der World Input-Output Datenbank (WIOD; Timmer et al. 2015), die die Verflechtungen zwischen je 56 Branchen $^{1}$ in 43 Ländern für die Jahre 2000 bis 2014 abbildet, zurückgegriffen. ${ }^{2}$ Basierend auf der Methodik von Johnson und Noguera (2012) und Koopman et al. (2014) kann ermittelt werden, wie stark Deutschland und Österreich in internationale Wertschöpfungsketten integriert sind und an welcher Position sich die beiden Ökonomien hier befinden. Im Anhang ist eine genaue Beschreibung der Methodik zur Berechnung der Partizipation und Position ergänzt.

Abb. 2 stellt die Wertschöpfungskettenpartizipation und -position einer Auswahl an Ökonomien dar. So sind Russland und Norwegen, zwei sehr rohstoffreiche Staaten, als stark ,upstream“ einzuordnen. Die Rohstoffe, die in Russland und Norwegen

\footnotetext{
1 Die Branchenklassifikation in der WIOD entspricht der ISIC Rev. 4 (siehe UN 2008).

2 Die WIOD ist eine von drei großen Datenbanken für weltweite Input-Output Tabellen, neben der OECD TiVA und der EORA. Die WIOD ist aus zweierlei Gründen zu präferieren. Zum einen ist die Branchenstruktur im Unterschied zu der OECD TiVA bei Produktions- und Dienstleistungsbranchen deutlich feingliedriger. Zum anderen bestehen bei der EORA, die in Hinblick auf die inkludierten Länder zu präferieren wäre, in der Literatur aber Bedenken bei der Datenqualität (vgl. Kowalski et al. 2015, S. 155).
} 
0,9

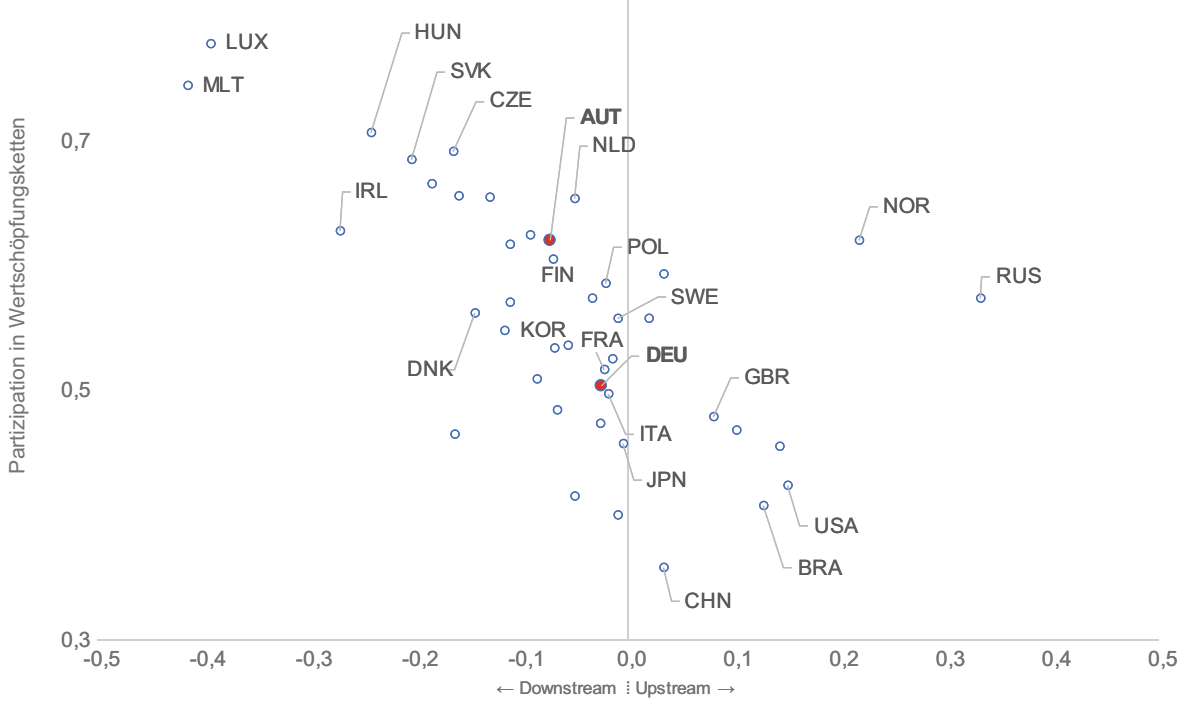

Abb. 2 Wertschöpfungskettenposition und -partizipation, 2014. (Die Partizipation in Wertschöpfungsketten wird durch das Ausmaß an ausländischen Vorleistungen in der Produktion der heimischen Bruttoexporte, sowie durch das Ausmaß an heimischen Vorleistungen in der Produktion der ausländischen Bruttoexporte definiert. Ob sich ein Land „,downstream“ oder „,upstream“ befindet, hängt vom Verhältnis der benötigten Vorleistungen zu dem Grad der Weiterverarbeitung heimischer Produkte ab. Eine genaue Beschreibung der dargestellten Indizes befindet sich im Anhang). (Quelle: eigene Berechnungen auf Basis der WIOD)

gewonnen werden, fließen als Vorleistungen in die Produktion vieler anderer Ökonomien mit ein. Am anderen Ende, d.h. sehr stark ,downstream“ rangieren Länder wie Luxemburg, Malta und Irland. Aber auch osteuropäische Staaten wie Ungarn, die Slowakei oder Tschechien sind tendenziell nahe am Endverbrauch. Dies können beispielsweise Finanzdienstleistungen oder verschiedenste Unternehmensdienstleistungen sein, aber auch der Vertrieb in Form der Logistik.

Deutschland und Österreich nehmen eine zentrale Rolle in globalen Wertschöpfungsketten ein, können also weder als stark „,upstream“, noch als stark ,downstream" beschrieben werden (vgl. Abb. 2). Österreich ist stärker in globale Wertschöpfungsketten integriert als Deutschland, wobei dies auch auf die Größe des Landes zurückzuführen ist. Tendenziell ist zu beobachten, dass größere Staaten (z.B. China oder USA) eine vergleichsweise geringe Wertschöpfungskettenpartizipation auf Basis der hier verwendeten Kennzahl aufweisen. ${ }^{3}$

\footnotetext{
3 Abb. 4 im Anhang stellt zudem die Wertschöpfungskettenpartizipation und -position für Deutschland und Österreich differenziert nach Branchen dar. Tendenziell sind Marktdienstleistungen in Deutschland und Österreich eher ,upstream“ situiert im Vergleich zur jeweiligen Position des Landes, während die Industrie eher „,downstream“ operiert.
} 


\section{Die Bedeutung ausländischer Endnachfrage für Deutschland und Österreich}

Die enge Einbindung in globale Wertschöpfungsketten und den Außenhandel im Allgemeinen hat zur Folge, dass die ausländische Endnachfrage (Investitionen und Konsum) für die Wertschöpfung im Inland immer bedeutender wird. Ein Endnachfrageschock in einem bestimmten Land hat zum einen direkten Einfluss auf die heimische Wirtschaft, indem die Exporte niedriger ausfallen. Zum anderen kommt es aber auch zu indirekten Effekten, da sich der Endnachfrageschock in diesem Land durch Wertschöpfungskettenverflechtungen auch über andere Handelspartner niederschlägt. Zum Beispiel: Kommt es in den USA zu einem beträchtlichen Nachfragerückgang betrifft das direkt exportierte Endprodukte aus Deutschland oder Österreich, die in den USA keinen Abnehmer zu gegebenen Preisen mehr finden. Darüber hinaus betrifft das aber auch den Export von Zwischengütern an einen dritten Handelspartner, der die Zwischengüter weiterverarbeitet und schließlich, nach einigen weiteren Produktionsschritten in weiteren Ländern, in den USA verkauft hätte. In der vorliegenden Analyse quantifizieren wir auf Basis der WIOD die Bedeutung der ausländischen Endnachfrage für die Wertschöpfung in Deutschland und Österreich.

Um diese Bedeutung zu messen, greifen wir auf das Konzept der Wertschöpfungsexporte nach Johnson und Noguera (2012) und Koopman et al. (2014) zurück. Wertschöpfungsexporte sind dabei definiert als die heimische Wertschöpfung, die schließlich im Ausland der Endnachfrage zugeführt wird. Werden die Wertschöpfungsexporte eines Landes der insgesamten heimischen Wertschöpfung gegenübergestellt, so stellt dieser Anteil die Bedeutung der ausländischen Endnachfragemärkte für die heimische Wirtschaft dar. Diese Methodik ist äquivalent zur Simulation eines Endnachfrageschocks in den einzelnen Partnerländern. Der Endnachfrageschock entspricht dabei einen Anstieg des Konsums und der Investitionen um ein Prozent über alle Branchen hinweg. Eine genaue Beschreibung der Methodik ist im Anhang verfügbar.

Es zeigt sich, dass die Bedeutung ausländischer Endnachfrage für beide Volkswirtschaften im Laufe der Zeit gestiegen ist: Im Jahr 2000 wurden rund 28,5\% an österreichischer Wertschöpfung im Ausland konsumiert oder investiert, 2014 waren dies bereits $33 \%$ der österreichischen Wertschöpfung. Für Deutschland ist die Veränderung im Zeitverlauf noch größer. Während im Jahr 2000 rund 24\% der deutschen Wertschöpfung im Ausland der Endnachfrage zugeführt wurden, stieg der Anteil bis 2014 auf 32,5\%. Sowohl in Deutschland als auch in Österreich wird somit rund ein Drittel der insgesamten Wertschöpfung der Endnachfrage im Ausland zugeführt. Damit liegen Deutschland und Österreich im Mittelfeld der in der WIOD abgebildeten Ökonomien (vgl. Abb. 5 im Anhang).

Weiters kann nach Partnerländern differenziert werden. Abb. 3 stellt die Bedeutung der zentralsten Endnachfragemärkte für Deutschland und Österreich in den Jahren 2000 und 2014 dar. Das Muster ist in beiden Staaten ähnlich: Die USA und China zählen zu den bedeutendsten Endnachfragemärkten für Deutschland und Österreich, gefolgt von Frankreich, Großbritannien und Italien. Österreich hängt hingegen zudem vor allem von deutscher Endnachfrage ab. In Bezug auf die Ver- 

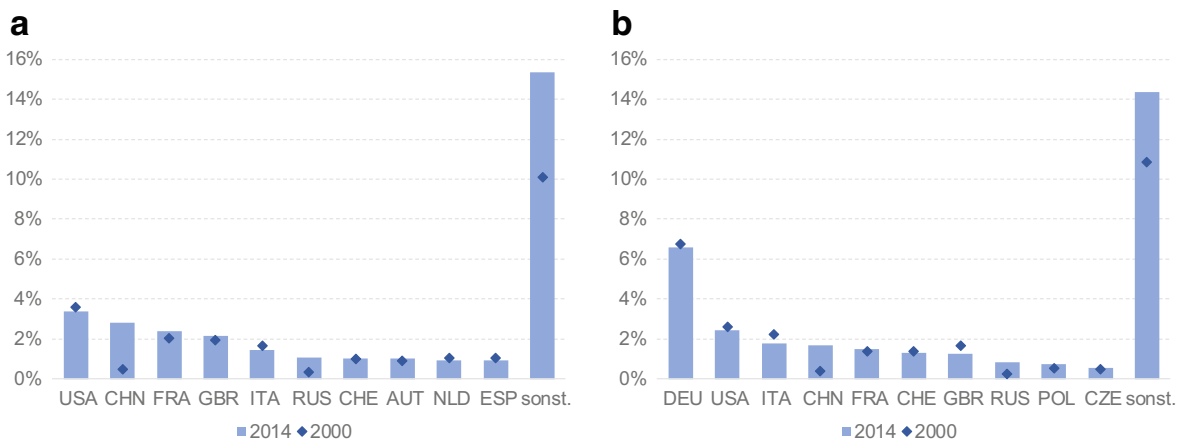

Abb. 3 Bedeutung einzelner Endnachfragemärkte für die Gesamtwirtschaft in Deutschland (a) und Österreich (b), 2000 \& 2014. (Quelle: eigene Berechnungen auf Basis der WIOD)

änderung über den Zeitraum von 2000 bis 2014 zeigt sich bei beiden Ländern eine stark zunehmende Bedeutung Chinas. Während im Jahr 2000 lediglich 0,48 $(0,38)$ Prozent der deutschen (österreichischen) Wertschöpfung in China der Endnachfrage zugeführt wurden, waren dies im Jahr 2014 bereits 2,8 (1,7) Prozent. Neben den zehn zentralsten Partnerländern ergibt sich eine relativ hohe Bedeutung sonstiger Staaten. Dies deutet in beiden Ländern auf eine diversifizierte Endnachfragestruktur hin und ist ein Zeichen dafür, dass sich die hier nicht explizit angeführten Staaten dynamischer entwickelten als klassische Märkte für deutsche und österreichische Wertschöpfung.

In einem weiteren Schritt kann auch zwischen der Bedeutung ausländischer Endnachfragemärkte auf der Ebene einzelner Branchen differenziert werden. Tab. 1 zeigt die Anteile der Wertschöpfung, die im Inland bzw. im Ausland konsumiert oder investiert werden, unterteilt nach einer groben Branchengliederung. Dabei wird deutlich, dass insbesondere deutsche und österreichische Wertschöpfung in Industriebranchen $\mathrm{zu}$ einem bedeutenden Teil der Endnachfrage im Ausland zugeführt werden. So beträgt dieser Anteil in Deutschland 70,9\%, in Österreich 77,4\%. Ähnlich verhält es sich in der Rohstoffgewinnung. 79,3\% der deutschen Wertschöpfung und 64,2\% der österreichischen Wertschöpfung in der Rohstoffgewinnung werden schließlich im Ausland konsumiert oder investiert. Bei Marktdienstleistungen ${ }^{4}$ zeigt sich ein gegensätzliches Bild. Der Großteil der Wertschöpfung in beiden Ökonomien (DE: 70,7\%, AT: 70,4\%) werden schließlich im Inland endnachgefragt. Auch in der Baubranche sowie der Energie- und Wasserversorgung bleibt mehr heimische Wertschöpfung bis zum Endverbrauch im Inland.

Auch gibt es aber Unterschiede zwischen Deutschland und Österreich. So ist die österreichische Land- und Forstwirtschaft stärker im Außenhandel involviert. Der Großteil (55,5\%) der österreichischen Wertschöpfung in dieser Branche fließt schließlich in die ausländische Endnachfrage. In Deutschland hingegen wird mit

\footnotetext{
${ }^{4}$ Hierunter sind alle Dienstleistungsbranchen außer öffentliche, Bildungs- und Gesundheitsdienstleistungen subsummiert.
} 
Tab. 1 Bedeutung der inländischen und ausländischen Endnachfrage einzelner Wirtschaftssektoren in Deutschland und Österreich, 2014. (Quelle: eigene Berechnungen auf Basis der WIOD)

\begin{tabular}{|c|c|c|c|c|c|c|c|c|}
\hline & & $\begin{array}{l}\text { Gesamt } \\
\text { in \% }\end{array}$ & $\begin{array}{l}\text { Land- } \\
\& \text { Forst- } \\
\text { wirtschaft } \\
\text { in } \%\end{array}$ & $\begin{array}{l}\text { Rohstoff- } \\
\text { gewin- } \\
\text { nung in } \\
\%\end{array}$ & $\begin{array}{l}\text { Industrie } \\
\text { in } \%\end{array}$ & $\begin{array}{l}\text { Energie- } \\
\& \text { Wasser- } \\
\text { versorgung } \\
\text { in } \%\end{array}$ & $\begin{array}{l}\text { Bau } \\
\text { in } \\
\%\end{array}$ & $\begin{array}{l}\text { Markt- } \\
\text { dienst- } \\
\text { leistun- } \\
\text { gen in } \\
\%\end{array}$ \\
\hline \multirow[t]{2}{*}{$\overline{\mathrm{DE}}$} & $\begin{array}{l}\text { Im Inland } \\
\text { der End- } \\
\text { nachfrage } \\
\text { zugeführt }\end{array}$ & 67,5 & 56,3 & 20,7 & 29,1 & 66,8 & 92,2 & 70,7 \\
\hline & $\begin{array}{l}\text { Im Ausland } \\
\text { der End- } \\
\text { nachfrage } \\
\text { zugeführt }\end{array}$ & 32,5 & 43,7 & 79,3 & 70,9 & 33,2 & 7,8 & 29,3 \\
\hline \multirow[t]{2}{*}{ AT } & $\begin{array}{l}\text { Im Inland } \\
\text { der End- } \\
\text { nachfrage } \\
\text { zugeführt }\end{array}$ & 67,0 & 44,5 & 35,8 & 22,6 & 55,1 & 89,0 & 70,4 \\
\hline & $\begin{array}{l}\text { Im Ausland } \\
\text { der End- } \\
\text { nachfrage } \\
\text { zugeführt }\end{array}$ & 33,0 & 55,5 & 64,2 & 77,4 & 44,9 & 11,0 & 29,6 \\
\hline
\end{tabular}

56,3\% der überwiegende Teil der Wertschöpfung in der Land- und Forstwirtschaft auch in Deutschland konsumiert oder investiert.

Darüber hinaus kann die Bedeutung der ausländischen Endnachfrage aber auch auf einer feingliedrigeren Branchenebene betrachtet werden. Tab. 3 im Anhang zeigt den Anteil der Wertschöpfung, der schließlich im Ausland konsumiert oder investiert wird, in 54 einzelnen Branchen, sowie den entsprechenden Anteil an der Wertschöpfung insgesamt. Dabei zeigt sich, dass die Bedeutung in einzelnen Industriebranchen die Werte aus Tab. 1 zum Teil deutlich übersteigt. So werden in Deutschland 76,2\% der Wertschöpfung der Kraftfahrzeugherstellung, die sich für rund $4 \%$ der insgesamten deutschen Wertschöpfung verantwortlich zeichnet, im Ausland der Endnachfrage zugeführt. In Österreich, wo die Kraftfahrzeugherstellung 1,3\% der Wertschöpfung ausmacht, werden sogar 91,3\% im Ausland konsumiert oder investiert. Gleichzeitig ergibt sich auch, dass einzelne Marktdienstleistungsbranchen in internationalen Wertschöpfungsketten eine bedeutende Rolle spielen und somit deren Wertschöpfung auch zu einem bedeutenden Teil im Ausland endnachgefragt wird. Dazu zählen der Großhandel, der in Deutschland (Österreich) 4,6 $(5,9) \%$ der insgesamten Wertschöpfung beiträgt und zu 48,4 (52,5) \% der ausländischen Endnachfrage zufließt, aber auch Finanzdienstleistungen oder die Werbung und Marktforschung.

\section{Wirtschaftspolitische Implikationen}

Die Ergebnisse dieses Beitrags zeigen, dass sowohl Deutschland als auch Österreich sehr eng in globale Wertschöpfungsketten eingebunden sind und die ausländische Endnachfrage dadurch direkt und indirekt für die heimische Wirtschaft im Allge- 
meinen und für einzelne Branchen im Speziellen bereits sehr bedeutend geworden ist. Rund ein Drittel der insgesamten Wertschöpfung Deutschlands und Österreichs fließt schließlich der Endnachfrage im Ausland zu. Dabei ist der Außenhandel insbesondere für Industriebranchen, in denen teilweise mehr als $80 \%$ der Wertschöpfung im Ausland konsumiert oder investiert werden, aber auch für einige Dienstleistungsbranchen wie den Großhandel oder Finanzdienstleistungen bedeutend.

Die tiefgreifende Integration in Wertschöpfungsketten hat vorwiegend positive Effekte auf die österreichische und deutsche Wirtschaft, da es erlaubt Effizienzgewinne im internationalen Wettbewerb zu erzielen und die positiven wirtschaftlichen Effekte des Freihandels zu generieren. So hat die Intensivierung des Freihandels und die Vertiefung der Wertschöpfungsketten über erhöhten Wettbewerb zu niedrigeren Preisen und Wohlfahrtsgewinnen geführt (vgl. Andrews et al. 2018; Felbermayr et al. 2018; Friesenbichler et al. 2021). Gleichzeitig führt ein höherer Grad an Handelsoffenheit aber durch eine stärkere Verschränkung der Volkswirtschaften und der vermehrte Handel von Intermediärgütern zu einer leichteren Verbreitung exogener, idiosynkratischer Schocks (Carvalho et al. 2020). Zudem kann es speziell bei der Herstellung komplexer Güter, die eine Reihe an hochspezialisierten Vorleistungen benötigen, über Produktionsverflechtungen zu einer Verstärkung von idiosynkratischen Schocks kommen, die schwer substituierbare Vorleistungen betreffen und damit die Herstellung des komplexen Guts verunmöglichen (Elliott et al. 2020).

Allerdings implizieren ein höherer Grad an Handelsoffenheit und damit einhergehende Wertschöpfungskettenverflechtungen auf einer makroökonomischen Ebene nicht zwangsweise eine höhere Volatilität der Wirtschaftsleistung. Zwar führt eine höhere Offenheit tendenziell zu einer stärkeren Spezialisierung der Wirtschaftsstruktur und kann damit aufgrund einer stärkeren Abhängigkeit von einzelnen Vorleistungen und Partnerländern die Volatilität erhöhen. Gleichzeitig reduziert sich durch die Möglichkeit Vorleistungs- und Absatzmärkte zu diversifizieren aber auch die Abhängigkeit der Wirtschaftsleistung von der heimischen Nachfrage und es kommt zu einer länderübergreifenden Angleichung der Konjunkturzyklen, das wiederum die Volatilität senken kann. Caselli et al. (2020) stellen diese Effekte gegenüber und zeigen, dass die zunehmende Globalisierung seit den 1970er-Jahren in zwei Drittel der Staaten, darunter auch Deutschland und Österreich, eine Stabilisierung des Wirtschaftswachstums bewirkt hat. Auch die COVID-19 Pandemie, die nicht als idiosynkratischer Schock sondern als weltweiter Wirtschaftseinbruch beschrieben werden kann, hätte in einer Welt ohne globalen Wertschöpfungskettenverflechtungen zu einem stärkeren Wirtschaftseinbruch geführt (Bonadio et al. 2020).

Darüber hinaus wäre eine Re-Regionalisierung und eine Rückkehr zu einer protektionistischeren Handelspolitik mit beachtlichen volkswirtschaftlichen Kosten verbunden. Kurzfristig kann dies zunächst zu Vergeltungsmaßnahmen anderer Handelspartner führen. Der Zollkrieg zwischen den USA und China der Jahre 2018-2020 zeigt dies eindrücklich (vgl. etwa Bown 2021). Der Ausgang eines Handelskriegs hängt in entscheidendem Maße von der Marktmacht der beteiligten Parteien im Welthandel ab, wie bereits Johnson (1953) argumentierte (vgl. hierzu auch Syropoulos 2002). Auch die im politischen Diskurs gestellte Forderung nach einer Re-Regionalisierung der internationalen Produktion kann reziproke Handlungen bei anderen 
Tab. 2 Anteil der Wertschöpfung in der EU, den USA und China, der im jeweiligen Ausland der Endnachfrage zugeführt wird. (Quelle: eigene Berechnungen auf Basis der WIOD)

\begin{tabular}{lllll}
\hline & & Exportiert nach & \\
& & EU & USA & CHN \\
\hline Exportiert von & EU & - & $2,6 \%$ & $1,6 \%$ \\
& USA & $2,0 \%$ & - & $0,7 \%$ \\
& CHN & $3,0 \%$ & $3,1 \%$ & - \\
\hline
\end{tabular}

Ländern auslösen, die dazu führen können, dass sich der Regionalisierungsprozess und die damit einhergehenden negativen Konsequenzen zusätzlich verstärken.

Vergeltungsmaßnahmen durch Handelspartner sind insofern ein relevanter Faktor, da Außenhandel per se auf Gegenseitigkeit beruht. Dies zeigt auch unsere Analyse auf Basis der WIOD (vgl. Tab. 2). So werden 2,6\% der Wertschöpfung der EU in den USA der Endnachfrage zugeführt, während rund 2,0\% der Wertschöpfung der USA in der EU der Endnachfrage zugeführt werden. Ein ähnlich reziprokes Bild zeigt sich zwischen der EU und China. Rund 1,6\% der Wertschöpfung der EU werden in China konsumiert oder investiert, während 3,0\% der chinesischen Wertschöpfung in der EU der Endnachfrage zufließen. Zwischen den USA und China zeigt sich ein etwas ungleicheres Bild: 0,7\% der US-amerikanischen Wertschöpfung werden in China der Endnachfrage zugeführt, während die USA als Endnachfragemarkt mit $3,1 \%$ der Wertschöpfung für China deutlich bedeutender ist.

Auch ohne die Gefahr einer reziproken Handlung von Handelspartnern hat Protektionismus im Kontext globaler Wertschöpfungsketten und Produktionsnetwerke schon negative Konsequenzen. So zeigen Barratieri und Cacciatore (2020), dass protektionistische Maßnahmen zwar einen geringen positiven Effekt auf die betroffenen Industrien haben, der Effekt auf die nachgelagerten Stufen der Produktionskette jedoch signifikant negativ und substantieller ist. Zudem sorgt die Integration in globale Wertschöpfungsketten für niedrigere Produktionskosten und damit niedrigere Preise für den Endnachfrager. Dies ist insbesondere auf einen stärkeren Wettbewerb in der Produktion zurückzuführen (Andrews et al. 2018). Eine Desintegration würde enstprechend zum Gegenteil führen. Vor dem Hintergrund, dass durch protektionistische Maßnahmen betroffene nachgelagerte Stufen und Endverbrauchermärkte auch im Ausland liegen, werden die Anreize für Vergeltungsmaßnahmen nochmal verstärkt.

Mittel- bis langfristig ist der Fokus auf eine Re-Regionalisierung zudem irreführend, da der internationale Kontext der Produktion ausgeblendet wird. Kurzfristig kann es zwar durchaus sein, dass hoch spezialisierte Produkte aus Deutschland oder Österreich aufgrund einer inelastischen Nachfrage auch trotz protektionistischer Maßnahmen und höheren Preisen im Ausland weiterhin nachgefragt werden. Mittelfristig kann das aber zu nicht intendierten Konsequenzen führen, indem andere Länder diese Chance nutzen und heimische Spezialisierungsvorteile verloren gehen können. Ein Beispiel hierfür ist die Entwicklung der deutschen Solarzellenproduktion. Vor 20 Jahren zählte Deutschland zu den führenden Herstellern von Photovoltaikanlagen. Mit dem Erneuerbare-Energien-Gesetz (EEG) von 2000 sollte, neben Investitionsbeihilfen in den neuen Bundesländern und Forschungsförderung 
in diesem Bereich, die heimische Produktion nachfrageseitig weiter stimuliert werden. Dies führte starken Anstieg bei der Errichtung von Photovoltaikanlagen (Wirth 2021). Die gestiegene Nachfrage konnte ab 2008/09 jedoch nicht mehr nur durch deutsche Produktion bedient werden. Dies rief andere Hersteller, vermehrt auch aus Asien und insbesondere China, auf den Plan, wo zur gleichen Zeit größere Produktionskapazitäten zur Solarzellenproduktion aufgebaut wurden, die aufgrund von positiven Skaleneffekten zunehmend wettbewerbsfähig wurden. Eine Untersuchung zur Entwicklung der globalen Solarzellenproduktion von Wen et al. (2020) zeigt, dass sich dieser Trend fortsetze und die Produktion in Deutschland relativ zu China stark abnahm und mittlerweile wenig Bedeutung hat.

Am aktuellen Rand ist in diesem Kontext die Produktion von Halbleitern, die in der Herstellung von technologischen Gütern essentiell sind, zu erwähnen. Bis dato waren hier insbesondere Produktionsverbünde zwischen Unternehmen in den USA, Japan, Taiwan, Korea und Europa federführend, die gegenüber chinesischen Herstellern einen technologischen Vorsprung von mehreren Jahren haben. Der Handelskrieg zwischen den USA und China hat seit 2019 zu einer Reihe Interventionen und Embargos in dieser Branche seitens der USA geführt, mit dem Ziel die Exporte nach China einzuschränken (siehe Bown 2020). China versucht nun mit weitreichenden Investitionen von mehr als $100 \mathrm{Mrd}$. Dollar so rasch wie möglich den technologischen Rückstand aufzuholen, um sich in hier selbstversorgen zu können (Economist 2021). Aus wirtschaftspolitischer Sicht ist es für die bisher in Halbleiter spezialisierten Regionen wie die USA und Europa zentral den noch bestehenden Technologievorsprung zumindest zu halten und möglichst auszubauen. Auf europäischer Ebene zählen Forschungsförderungen im Rahmen der Important Projects of Common European Interest (IPCEI), die innerhalb der EU länderübergreifende Unternehmenskooperationen in zukünftig zentralen Wertschöpfungsketten (u.a. auch Mikroelektronik bzw. Halbleiter) unterstützen sollen, zu den bereits gesetzten Maßnahmen. Ein wirtschaftspolitischer Fokus auf Re-Regionalisierung in diesem Bereich kann für die in dieser Branche tätigen Unternehmen mittel- bis langfristig zu einer ähnlichen Entwicklung wie jene der deutschen Solarzellenproduzenten führen.

Die COVID-19 Pandemie sollte aus den angeführten Gründen nicht als Anlass herangezogen werden, um internationale Wertschöpfungsketten zu re-regionalisieren, da eine Rückkehr zu einer protektionistischeren Handelspolitik mit beachtlichen volkswirtschaftlichen Kosten verbunden wäre. Eine Neuorientierung würde eine Bewegung weg von den derzeitigen Stärken und Spezialisierungen der deutschen und österreichischen Volkswirtschaften bedeuten und damit einen Verlust von firmenspezifischen immateriellen Vermögenswerten wie Patenten sowie Know-How und schließlich auch an Wettbewerbsfähigkeit mit sich bringen.

Ziel muss es daher sein, die Position österreichischer und deutscher Unternehmen in globalen Wertschöpfungsketten und im Außenhandel durch eine Steigerung der Produktivität weiter zu stärken. Wirtschaftspolitische Ansatzpunkte hierfür sind unter anderem Qualitätsverbesserungen im Bildungsbereich, der Ausbau digitaler Infrastruktur, sowie eine gesamtheitlich wettbewerbsfähige Standortpolitik.

Letztendlich gilt es auch die Entwicklung der europäischen und weltweiten Integration weiter mitzugestalten. Der Sachverständigenrat (2020, S. 193ff.) hat hierzu 
im aktuellen Jahresgutachten unter anderem die Empfehlung ausgesprochen, die noch immer vorhandenen Barrieren im Dienstleistungshandel innerhalb des EUBinnenmarkts zu reduzieren, sowie einen freieren Kapital- und Zahlungsverkehr zu ermöglichen. Zudem können sich Deutschland und Österreich auf Ebene der Europäischen Union für den Erhalt der Welthandelsorganisation sowie für den Abschluss bi- und multilateraler Freihandelsabkommen einsetzen, die das einzige Instrument der EU darstellen, um gegen die Erosion der Rechtssicherheit im internationalen Handel vorzugehen.

\section{Anhang}

Die generelle Struktur einer Input-Output Tabelle mit $S$ Branchen pro Land und $C$ Ländern (d.h. $N=S^{*} C$ insgesamten Branchen), wobei $\mathbf{X}$ den Output pro Branche beschreibt, kann als $\mathbf{X}=\mathbf{A X}+\mathbf{Y}$ definiert werden. Dies ist gleichbedeutend mit

$$
\boldsymbol{X}=(\boldsymbol{I}-\boldsymbol{A})^{-1} \boldsymbol{Y}
$$

Der Vektor Y beschreibt dabei die Endnachfrage, während die Matrix A die Input-Output Koeffizienten darstellt. Die Matrix $(\mathbf{I}-\mathbf{A})^{-1}$ ist die sogenannte LeontiefInverse, wobei das Element $\left([\mathbf{I}-\mathbf{A}]^{-1}\right)_{\mathrm{ij}}$ beschreibt, wie viele Einheiten vom Intermediärgut der Branche $x_{i}$ benötigt werden, um eine Einheit eines Endprodukts in Branche $y_{j}$ zu produzieren.

Auf Basis der Methodik von Johnson und Noguera (2012) und Koopman et al. (2014) definieren wir für die Berechnung der Wertschöpfung auf Branchenebene die Diagonalmatrix $\mathbf{V}$, die Wertschöpfungskoeffizienten darstellt, sodass

$$
[\boldsymbol{V}]_{i}=[\boldsymbol{I}]_{i}-\sum_{n=1}^{N}[\boldsymbol{A}]_{n i}
$$

Die Wertschöpfung einzelner Branchen sowie deren Endnachfragemarkt können anschließend der Matrix VA entnommen werden, die definiert ist als

$$
\boldsymbol{V A}=\boldsymbol{V} *(\boldsymbol{I}-\boldsymbol{A})^{-1} * \boldsymbol{Y}
$$

Dabei beschreibt das Element $[\mathbf{V A}]_{\mathrm{ij}}$ die Wertschöpfung, die von Branche $i$ nach Land $j$ fließt. Die Diagonalblockmatrizen stellen die Wertschöpfung dar, die der inländischen Endnachfrage zugeführt wird. Die Summe der Zeile $i$ stellt die insgesamte Wertschöpfung der Branche $i$ dar.

Auf Basis der Matrix VA kann die Bedeutung ausländischer Endnachfragemärkte für die heimische Wertschöpfung abgeschätzt werden. Diese Methodik ist äquivalent zu einer Simulation eines branchenübergreifenden Endnachfrageschocks (Konsum und Investitionen) in einzelnen Partnerländern um $1 \%$.

Zur Quantifizierung der Vorleistungs- und Weiterverarbeitungsverflechtungen, die die Position und Partizipation in globalen Wertschöpfungsketten determinieren, wird 
die Matrix T definiert. Diese ist das Produkt der Matrizen V (siehe oben), der Leontief-Inversen (siehe oben), und dem Vektor der Bruttoexporte E, d.h.

$$
\boldsymbol{T}=\boldsymbol{V} *(\boldsymbol{I}-\boldsymbol{A})^{-1} * \boldsymbol{E}
$$

Betrachtet man die Matrix $\mathbf{T}$ in Form von Blockmatrizen, die einzelne Länder beschreiben, so stellt die Spalte $i$ dar, welche Vorleistungen (gemessen an der Wertschöpfung) in die Bruttoexporte des Landes $i$ einfließen. Dabei wird unterteilt zwischen heimischer Wertschöpfung (,domestic value-added“, DVA), die in den diagonalen Einträgen zu finden ist, und ausländischer Wertschöpfung (,foreign valueadded“, FVA). Die Methodik erlaubt nach einzelnen Ländern und Branchen zu unterscheiden. Darüber hinaus beschreibt die Zeile $j$ der Matrix $\mathbf{T}$ in welchen Ländern und Branchen Wertschöpfung aus Land $j$ als Vorleistung für die Exporte anderer Länder dient (,indirect domestic value-added“, DVX).

Um die Partizipation in Wertschöpfungsketten zu bestimmen, wird die Kennzahl $G V C_{\text {part }}$ (,global value chain participation“) herangezogen (Aslam et al. 2017):

$$
\mathrm{GVC}_{\text {part }}=\frac{F V A+D V X}{\text { Bruttoexporte }}
$$

Zur Berechnung der Position in Wertschöpfungsketten wird der $R U$-Index (,relative upstreamness“) von Koopman et al. (2014) herangezogen:

$$
R U=\ln \left(1+\frac{D V X}{\text { Bruttoexporte }}\right)-\ln \left(1+\frac{F V A}{\text { Bruttoexporte }}\right)
$$




\section{a}

a

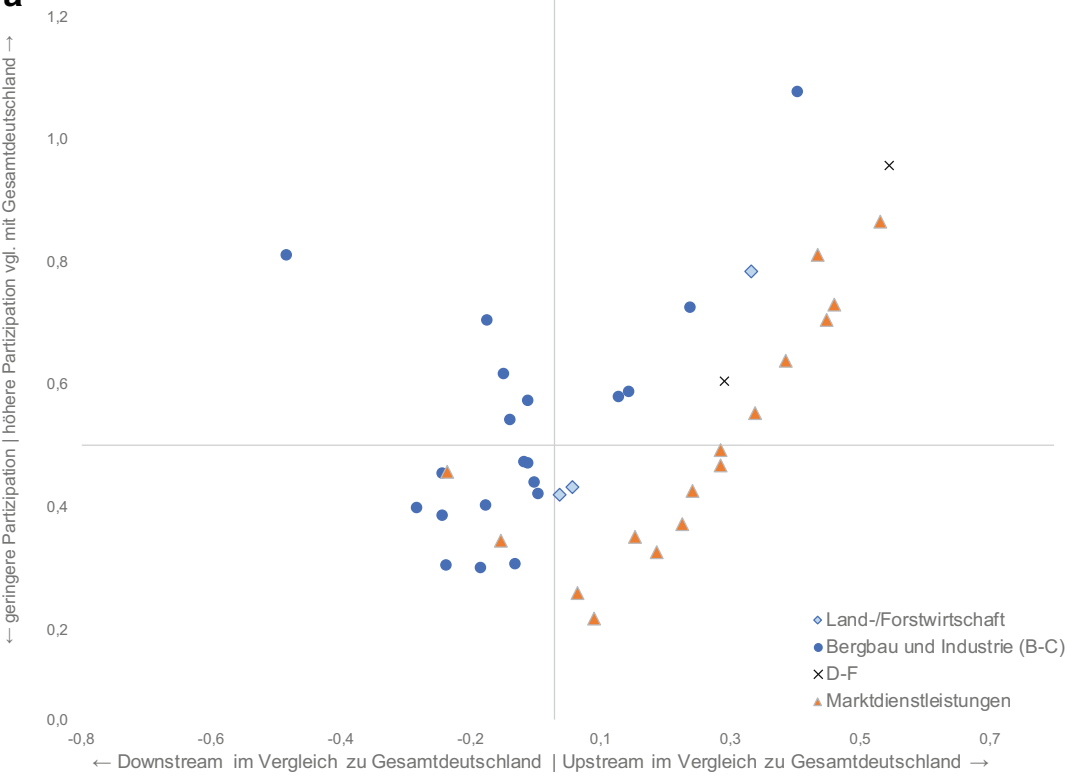

b

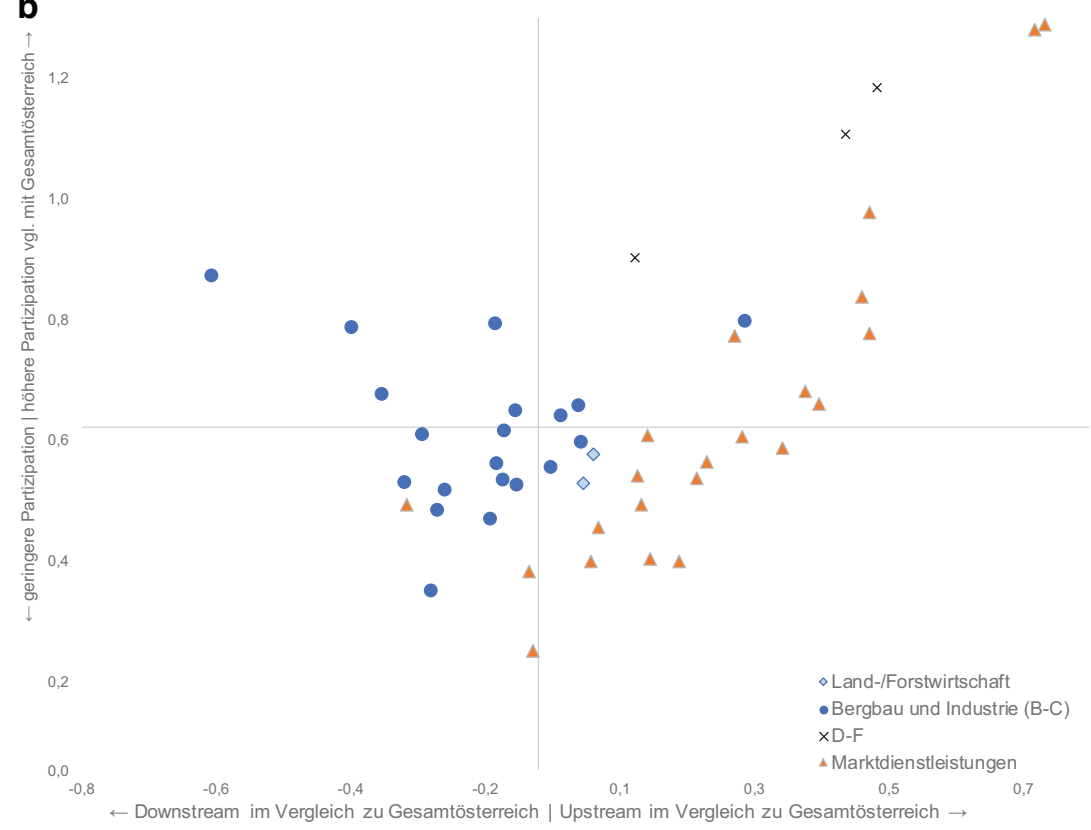

Abb. 4 Wertschöpfungskettenposition und -partizipation auf Branchenebene für Deutschland (a) und Österreich (b), 2014. (Quelle: eigene Berechnungen auf Basis der WIOD) 


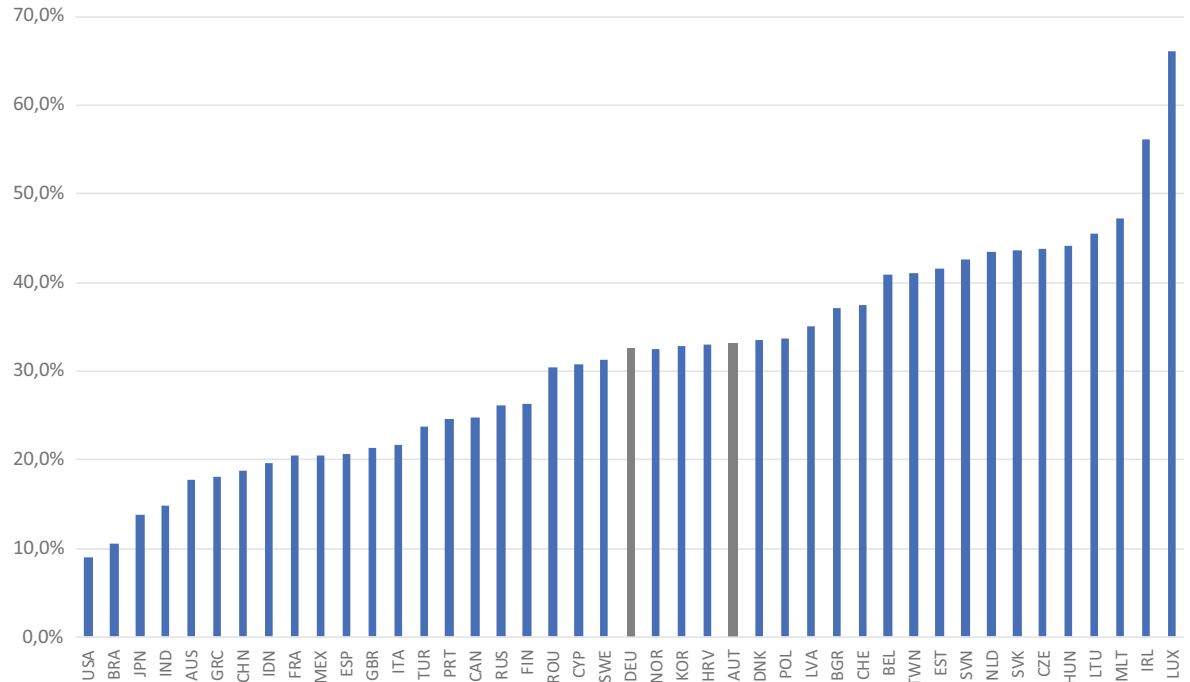

Abb. 5 Anteil der im Ausland der Endnachfrage zugeführten Wertschöpfung, 2014. (Quelle: eigene Berechnungen auf Basis der WIOD)

Tab. 3 Bedeutung ausländischer Endnachfrage auf Branchenebene, 2014. (Quelle: eigene Berechnungen auf Basis der WIOD)

\begin{tabular}{|c|c|c|c|c|c|}
\hline \multirow{2}{*}{$\begin{array}{l}\text { ISIC- } \\
\text { Code }\end{array}$} & \multicolumn{3}{|c|}{ Kurzbezeichnung Deutschland } & \multicolumn{2}{|l|}{ Österreich } \\
\hline & & $\begin{array}{l}\text { Anteil an } \\
\text { Wertschöp- } \\
\text { fung ins- } \\
\text { gesamt in } \\
\%\end{array}$ & $\begin{array}{l}\text { Schließlich im } \\
\text { Ausland konsu- } \\
\text { miert o. investiert } \\
\text { in } \%\end{array}$ & $\begin{array}{l}\text { Anteil an } \\
\text { Wertschöp- } \\
\text { fung ins- } \\
\text { gesamt in } \\
\%\end{array}$ & $\begin{array}{l}\text { Schließlich im } \\
\text { Ausland kon- } \\
\text { sumiert oder } \\
\text { investiert in \% }\end{array}$ \\
\hline A01 & $\begin{array}{l}\text { Land- und } \\
\text { Tierwirtschaft }\end{array}$ & 0,6 & 44,7 & 1,0 & 57,5 \\
\hline $\mathrm{A} 02$ & Forstwirtschaft & 0,1 & 34,1 & 0,4 & 51,4 \\
\hline A03 & Fischerei & 0,0 & 65,8 & 0,0 & 18,2 \\
\hline B & Bergbau & 0,2 & 79,3 & 0,5 & 64,2 \\
\hline $\mathrm{C} 10-\mathrm{C} 12$ & Nahrungsmittel & 1,6 & 38,7 & 1,9 & 58,2 \\
\hline $\mathrm{C} 13-\mathrm{C} 15$ & Textilproduktion & 0,3 & 91,6 & 0,4 & 94,5 \\
\hline $\mathrm{C} 16$ & $\begin{array}{l}\text { Holzproduktion } \\
\text { (ohne Möbel) }\end{array}$ & 0,2 & 44,5 & 0,7 & 60,3 \\
\hline $\mathrm{C} 17$ & $\begin{array}{l}\text { Papier und } \\
\text { Pappe }\end{array}$ & 0,4 & 71,5 & 0,6 & 84,8 \\
\hline $\mathrm{C} 18$ & $\begin{array}{l}\text { Verlag und } \\
\text { Druck }\end{array}$ & 0,3 & 37,1 & 0,3 & 58,5 \\
\hline C19 & Raffinerie & 0,2 & 44,5 & 0,0 & 57,3 \\
\hline $\mathrm{C} 20$ & $\begin{array}{l}\text { Chemische } \\
\text { Produkte }\end{array}$ & 1,6 & 82,1 & 0,7 & 90,8 \\
\hline $\mathrm{C} 21$ & $\begin{array}{l}\text { Pharmazeutische } \\
\text { Prod }\end{array}$ & 0,9 & 83,7 & 0,7 & 78,7 \\
\hline $\mathrm{C} 22$ & $\begin{array}{l}\text { Gummi und } \\
\text { Plastik }\end{array}$ & 1,0 & 71,2 & 0,8 & 80,2 \\
\hline
\end{tabular}


Tab. 3 (Fortsetzung)

\begin{tabular}{|c|c|c|c|c|c|}
\hline \multirow{2}{*}{$\begin{array}{l}\text { ISIC- } \\
\text { Code }\end{array}$} & \multicolumn{3}{|c|}{ Kurzbezeichnung Deutschland } & \multicolumn{2}{|l|}{ Österreich } \\
\hline & & $\begin{array}{l}\text { Anteil an } \\
\text { Wertschöp- } \\
\text { fung ins- } \\
\text { gesamt in } \\
\%\end{array}$ & $\begin{array}{l}\text { Schließlich im } \\
\text { Ausland konsu- } \\
\text { miert o. investiert } \\
\text { in } \%\end{array}$ & $\begin{array}{l}\text { Anteil an } \\
\text { Wertschöp- } \\
\text { fung ins- } \\
\text { gesamt in } \\
\%\end{array}$ & $\begin{array}{l}\text { Schließlich im } \\
\text { Ausland kon- } \\
\text { sumiert oder } \\
\text { investiert in \% }\end{array}$ \\
\hline $\mathrm{C} 23$ & $\begin{array}{l}\text { Sonst. nicht- } \\
\text { metall. Pro- } \\
\text { dukte }\end{array}$ & 0,6 & 45,9 & 0,9 & 53,8 \\
\hline $\mathrm{C} 24$ & $\begin{array}{l}\text { Metallerzeugung } \\
\text { und -bearbei- } \\
\text { tung }\end{array}$ & 0,8 & 77,5 & 1,3 & 85,5 \\
\hline $\mathrm{C} 25$ & $\begin{array}{l}\text { Metaller- } \\
\text { zeugnisse }\end{array}$ & 2,1 & 61,4 & 1,9 & 77,2 \\
\hline $\mathrm{C} 26$ & $\begin{array}{l}\text { Computer, } \\
\text { Elektronik } \\
\text { und Optik }\end{array}$ & 1,3 & 87,2 & 1,0 & 86,3 \\
\hline $\mathrm{C} 27$ & Elektrogeräte & 1,7 & 77,4 & 1,5 & 83,8 \\
\hline $\mathrm{C} 28$ & $\begin{array}{l}\text { Sonst. Ma- } \\
\text { schinenbau }\end{array}$ & 3,6 & 77,1 & 2,6 & 86,3 \\
\hline $\mathrm{C} 29$ & Kraftfahrzeuge & 4,0 & 76,2 & 1,3 & 91,3 \\
\hline $\mathrm{C} 30$ & $\begin{array}{l}\text { Sonst. Fahr- } \\
\text { zeugbau }\end{array}$ & 0,5 & 99,5 & 0,3 & 89,1 \\
\hline C31_C32 & $\begin{array}{l}\text { Möbel u. } \\
\text { andere Er- } \\
\text { zeugnisse }\end{array}$ & 0,9 & 65,9 & 0,8 & 73,2 \\
\hline $\mathrm{C} 33$ & $\begin{array}{l}\text { Rep. u. Inst. } \\
\text { v. Maschinen }\end{array}$ & 0,6 & 42,4 & 0,8 & 55,7 \\
\hline D35 & $\begin{array}{l}\text { Energie- } \\
\text { versorgung }\end{array}$ & 1,9 & 26,6 & 1,7 & 44,0 \\
\hline E36 & $\begin{array}{l}\text { Wasser- } \\
\text { versorgung }\end{array}$ & 0,2 & 23,3 & 0,2 & 22,1 \\
\hline E37-E39 & $\begin{array}{l}\text { Abwasser, } \\
\text { Abfall, Ent- } \\
\text { sorgung }\end{array}$ & 0,8 & 50,8 & 0,9 & 51,7 \\
\hline $\mathrm{F}$ & Baugewerbe & 4,6 & 7,8 & 6,4 & 11,0 \\
\hline G45 & $\begin{array}{l}\text { Groß-/ } \\
\text { Einzelhandel } \\
\text { v. Fahrzeugen }\end{array}$ & 1,5 & 34,9 & 1,5 & 24,7 \\
\hline G46 & Großhandel & 4,6 & 48,4 & 5,9 & 52,5 \\
\hline G47 & Einzelhandel & 3,2 & 12,1 & 4,7 & 6,6 \\
\hline H49 & Landtransport & 1,9 & 36,8 & 2,9 & 54,5 \\
\hline H50 & Wassertransport & 0,3 & 97,2 & 0,0 & 88,8 \\
\hline H51 & Lufttransport & 0,2 & 32,6 & 0,2 & 44,5 \\
\hline H52 & $\begin{array}{l}\text { Lagerei und } \\
\text { sonst. Ver- } \\
\text { kehrs-DL }\end{array}$ & 1,8 & 53,0 & 2,1 & 50,8 \\
\hline H53 & $\begin{array}{l}\text { Post- und } \\
\text { Kurierdienste }\end{array}$ & 0,5 & 34,8 & 0,5 & 42,7 \\
\hline
\end{tabular}


Tab. 3 (Fortsetzung)

\begin{tabular}{|c|c|c|c|c|c|}
\hline \multirow{2}{*}{$\begin{array}{l}\text { ISIC- } \\
\text { Code }\end{array}$} & \multicolumn{3}{|c|}{ Kurzbezeichnung Deutschland } & \multicolumn{2}{|l|}{ Österreich } \\
\hline & & $\begin{array}{l}\text { Anteil an } \\
\text { Wertschöp- } \\
\text { fung ins- } \\
\text { gesamt in } \\
\%\end{array}$ & $\begin{array}{l}\text { Schließlich im } \\
\text { Ausland konsu- } \\
\text { miert o. investiert } \\
\text { in } \%\end{array}$ & $\begin{array}{l}\text { Anteil an } \\
\text { Wertschöp- } \\
\text { fung ins- } \\
\text { gesamt in } \\
\%\end{array}$ & $\begin{array}{l}\text { Schließlich im } \\
\text { Ausland kon- } \\
\text { sumiert oder } \\
\text { investiert in \% }\end{array}$ \\
\hline I & $\begin{array}{l}\text { Beherbergung- } \\
\text { und Gastro- } \\
\text { nomie }\end{array}$ & 1,5 & 11,0 & 5,2 & 14,3 \\
\hline $\mathrm{J} 58$ & Verlagswesen & 0,6 & 32,1 & 0,3 & 48,0 \\
\hline J59_J60 & $\begin{array}{l}\text { Hst. v. Fil- } \\
\text { men, Videos, } \\
\text { Musik }\end{array}$ & 0,7 & 25,4 & 0,4 & 44,7 \\
\hline J61 & $\begin{array}{l}\text { Telekommuni- } \\
\text { kation }\end{array}$ & 1,0 & 19,4 & 0,8 & 29,8 \\
\hline J62_J63 & $\begin{array}{l}\text { Informations- } \\
\text { technologie }\end{array}$ & 2,6 & 40,3 & 1,8 & 39,2 \\
\hline K64 & $\begin{array}{l}\text { Finanzdienst- } \\
\text { leistungen }\end{array}$ & 2,5 & 29,2 & 3,2 & 39,1 \\
\hline K65 & Versicherungen & 1,0 & 19,6 & 1,0 & 22,8 \\
\hline K66 & $\begin{array}{l}\text { Sonst. } \\
\text { Dienstleis- } \\
\text { tungen im } \\
\text { Finanzbe- } \\
\text { reich }\end{array}$ & 0,6 & 21,7 & 0,4 & 29,4 \\
\hline L68 & $\begin{array}{l}\text { Grundstücks- } \\
\text { und Woh- } \\
\text { nungswesen }\end{array}$ & 11,2 & 10,8 & 9,9 & 9,4 \\
\hline M69_70 & $\begin{array}{l}\text { Unternehmens- } \\
\text { beratung, } \\
\text { Headquarters }\end{array}$ & 3,0 & 46,8 & 2,7 & 44,7 \\
\hline M71 & $\begin{array}{l}\text { Architektur- } \\
\text { und Inge- } \\
\text { nieurbüros }\end{array}$ & 1,5 & 40,7 & 1,5 & 37,5 \\
\hline M72 & $\begin{array}{l}\text { Forschung \& } \\
\text { Entwicklung }\end{array}$ & 0,8 & 17,8 & 0,4 & 23,4 \\
\hline M73 & $\begin{array}{l}\text { Werbung und } \\
\text { Marktfor- } \\
\text { schung }\end{array}$ & 0,5 & 55,6 & 0,4 & 56,2 \\
\hline M74_M75 & $\begin{array}{l}\text { Sonst. Frei- } \\
\text { berufliche } \\
\text { Dienstleistun- } \\
\text { gen }\end{array}$ & 0,5 & 37,3 & 0,2 & 37,2 \\
\hline $\mathrm{N}$ & $\begin{array}{l}\text { Administrative } \\
\text { Dienstleistun- } \\
\text { gen }\end{array}$ & 4,9 & 36,2 & 4,3 & 33,2 \\
\hline
\end{tabular}


Tab. 3 (Fortsetzung)

\begin{tabular}{|c|c|c|c|c|c|}
\hline \multirow{2}{*}{$\begin{array}{l}\text { ISIC- } \\
\text { Code }\end{array}$} & \multicolumn{3}{|c|}{ Kurzbezeichnung Deutschland } & \multicolumn{2}{|l|}{ Österreich } \\
\hline & & $\begin{array}{l}\text { Anteil an } \\
\text { Wertschöp- } \\
\text { fung ins- } \\
\text { gesamt in } \\
\%\end{array}$ & $\begin{array}{l}\text { Schließlich im } \\
\text { Ausland konsu- } \\
\text { miert o. investiert } \\
\text { in } \%\end{array}$ & $\begin{array}{l}\text { Anteil an } \\
\text { Wertschöp- } \\
\text { fung ins- } \\
\text { gesamt in } \\
\%\end{array}$ & $\begin{array}{l}\text { Schließlich im } \\
\text { Ausland kon- } \\
\text { sumiert oder } \\
\text { investiert in \% }\end{array}$ \\
\hline$\overline{\mathrm{O} 84}$ & $\begin{array}{l}\text { Öffentliche } \\
\text { Verwaltung } \\
\text { und Verteidi- } \\
\text { gun }\end{array}$ & 6,2 & 6,1 & 5,2 & 2,4 \\
\hline P85 & Bildung & 4,5 & 3,1 & 5,4 & 4,0 \\
\hline Q & $\begin{array}{l}\text { Gesundheits- } \\
\text { dienstleistungen }\end{array}$ & 7,6 & 0,4 & 6,8 & 0,9 \\
\hline R_S & $\begin{array}{l}\text { Sonst. } \\
\text { Dienstleis- } \\
\text { tungen }\end{array}$ & 3,8 & 6,9 & 2,8 & 8,0 \\
\hline
\end{tabular}

Danksagung Wir bedanken uns bei einem/einer anonymen Reviewer/in und Monika Köppl-Turyna für hilfreiche Anmerkungen.

\section{Literatur}

Andrews, D., Gal, P., \& Witheridge, W. (2018). A genie in a bottle? Globalisation, competition and inflation. OECD Economics Department working paper no. 1462.

Aslam, A., Novta, N., \& Rodrigues-Bastos, F. (2017). Calculating trade in value added. IMF working paper WP/17/178.

Barratieri, A., \& Cacciatore, M. (2020). Self-harming trade policy? Protectionism and production networks. NBER working paper 27630. https://doi.org/10.3386/w27630.

Berger, J., Graf, N., Strohner, L., \& Schuh, U. (2014). Wirtschaftliche Auswirkungen der österreichischen Mitgliedschaft in der Europäischen Union. EcoAustria Policy Note 7.

Bonadio, B., Huo, Z., Levchenko, A. A., \& Pandalai-Nayar, N. (2020). Global supply chains in the pandemic. NBER working paper 27224.

Bown, C.P. (2020). How the United States marched the semiconductor industry into its trade war with China. PIIE Working Paper No. 20. 16. Dezember

Bown, C.P. (2021). The US-China trade war and phase one agreement. PIIE working paper no. 21. 2. März

Breuss, F. (2020). Makroökonomische Effekte der 25-jährigen EU-Mitgliedschaft Österreichs. Monetary Policy \& the Economy. Quarterly Review of Economic Policy, Q1-Q2/20, 27-28.

Carvalho, V.M., Nirei, M., Saito, Y.U., \& Tahbaz-Salehi, A. (2020). Supply chain disruptions: evidence from the great East Japan earthquake. The Quarterly Journal of Economics. https://doi.org/10.1093/ qje/qjaa044.

Caselli, F., Koren, M., Lisicky, M., \& Tenreyro, S. (2020). Diversification through trade. The Quarterly Journal of Economics, 135(1), 449-502.

Economist (2021). Technology and geopolitics: the struggle over chips enters a new phase. https://econ.st/ 3qZjchO. Zugegriffen: 17. März 2021.

Elliott, M., Golub, B., \& Leduc, M. V. (2020). Supply network formation and fragility. Cambridge working paper economics, 2028.

Felbermayr, G., \& Görg, H. (2020). Die Folgen von Covid-19 für die Globalisierung. Perspektiven der Wirtschaftspolitik, 21(3), 263-272. https://doi.org/10.1515/pwp-2020-0025.

Felbermayr, G., Gröschl, J., \& Heiland, I. (2018). Undoing europe in a new quantitative trade model. ifo working paper 250 . 
Fiorini, M., Hoekman, B., \& Yildirm, A. (2020). COVID-19: expanding access to essential supplies in a value chain world. In R.E. Baldwin \& S. J. Evenett (Hrsg.), COVID-19 and trade policy: why turning inward won't work (S. 63-76). London: CEPR Press.

Flach, L., Aichele, R., \& Braml, M. (2020). Status quo und Zukunft globaler Lieferketten. ifo Schnelldienst, 73(5), 16-22.

Friesenbichler, K. S., Kuegler, A., \& Reinstaller, A. (2021). Does value chain integration dampen producer price developments? Evidence from the European Union. The World Economy, 44, 89-106. https:// doi.org/10.1111/twec. 12993 .

IBRD (2017). Measuring and analyzing the impact of GVCs on economic development. Washington D.C.: World Bank.

Johnson, H. G. (1953). Optimum tariffs and retaliation. The Review of Economic Studies, 21(2), 142-153.

Johnson, R.C., \& Noguera, G. (2012). Accounting for intermediates: production sharing and trade in value added. Journal of International Economics, 86(2), 224-236. https://doi.org/10.1016/j.jinteco.2011. 10.003 .

Koopman, R., Wang, Z., \& Wei, S.-J. (2014). Tracing value-added and double counting in gross exports. American Economic Review, 104(2), 459-494.

Kowalski, P., Gonzalez, J. L., Ragoussis, A., \& Ugarte, C. (2015). Participation of developing countries in global value chains: implications for trade and trade-related policies. OECD Trade Policy Papers, Bd. 179. Paris: OECD Publishing.

Mayer, T., Vicard, V., \& Zignago, S. (2019). The cost of non-Europe, revisited. Economic Policy, 34(98), 145-199.

Sachs, E., Funke, C., Kreuzer, P., \& Weiss, J. (2020). Globalisierungsreport 2020. Wer profitiert am stärksten von der Globalisierung? Gütersloh: Bertelsmann Stiftung.

Sachverständigenrat (2020). Corona-Krise gemeinsam bewältigen, Resilienz und Wachstum stärken: Jahresgutachten 2020/21

Stehrer, R., \& Stöllinger, R. (2015). The central European manufacturing core: what is driving regional production sharing? FIW-research reports, Bd. 2014/15 $\mathrm{N}^{\circ} 02$.

Syropoulos, C. (2002). Optimum tariffs and retaliation revisited: how country size matters. The Review of Economic Studies, 69(3), 707-727. https://doi.org/10.1111/1467-937X.t01-1-00028.

Timmer, M. P., Dietzenbacher, E., Los, B., Stehrer, R., \& de Vries, G. J. (2015). An illustrated user guide to the world input-output database: the case of global automotive production. Review of International Economics, 23, 575-605.

UN (2008). International standard industrial classification of all economic activities, revision 4 . New York: United Nations.

Weltbank (2019). World development report 2020: trading for development in the age of global value chains. Washington, DC: World Bank Group.

Wen, D., Gao, W., Qian, F., Gu, Q., \& Ren, J. (2020). Development of solar photovoltaic industry and market in China, Germany, Japan and the United States of America using incentive policies. Energy Exploration \& Exploitation. https://doi.org/10.1177/0144598720979256.

Wirth, H. (2021). Aktuelle Fakten zur Photovoltaik in Deutschland. www.pv-fakten.de. Zugegriffen: 11. März 2021. Fraunhofer ISE. 